\title{
Expression and Functional Roles of the Pepper Pathogen-Induced bZIP Transcription Factor CabZIP2 in Enhanced Disease Resistance to Bacterial Pathogen Infection
}

\author{
Chae Woo Lim, ${ }^{1}$ Woonhee Baek, ${ }^{1}$ Sohee Lim, ${ }^{1}$ Sang-Wook Han, ${ }^{2}$ and Sung Chul Lee ${ }^{1}$ \\ ${ }^{1}$ Department of Life Science (BK21 program), Chung-Ang University, Seoul, 156-756, Republic of Korea; ${ }^{2}$ Department of \\ Integrative Plant Science, Chung-Ang University, Anseong, 456-756, Republic of Korea
}

Submitted 9 October 2015. Accepted 10 February 2015.

\begin{abstract}
A pepper bZIP transcription factor gene, $\mathrm{CabZIP2}$, was isolated from pepper leaves infected with a virulent strain of Xanthomonas campestris pv. vesicatoria. Transient expression analysis of the CabZIP2-GFP fusion protein in Nicotiana benthamiana revealed that the CabZIP2 protein is localized in the cytoplasm as well as the nucleus. The acidic domain in the $\mathrm{N}$-terminal region of CabZIP2 that is fused to the GAL4 DNA-binding domain is required to activate the transcription of reporter genes in yeast. Transcription of CabZIP2 is induced in pepper plants inoculated with virulent or avirulent strains of $X$. campestris pv. vesicatoria. The CabZIP2 gene is also induced by defense-related hormones such as salicylic acid, methyl jasmonate, and ethylene. To elucidate the in vivo function of the CabZIP2 gene in plant defense, virus-induced gene silencing in pepper and overexpression in Arabidopsis were used. CabZIP2-silenced pepper plants were susceptible to infection by the virulent strain of $X$. campestris pv. vesicatoria, which was accompanied by reduced expression of defense-related genes such as CaBPRI and CaAMP1. CabZIP2 overexpression in transgenic Arabidopsis plants conferred enhanced resistance to Pseudomonas syringae pv. tomato DC3000. Together, these results suggest that CabZIP2 is involved in bacterial disease resistance.
\end{abstract}

As plants are sessile organisms, they are constantly contiguous to unfavorable environmental conditions, such as drought, high salinity, and pathogens. Plants defend themselves against various pathogens, including bacteria, fungi, viruses, and nematodes. The activation of defense mechanisms is initiated by the recognition of pathogen associated molecular patterns (PAMPs) by pattern recognition receptors (Jones and Dangl 2006). Pathogen recognition by plants activates signal transduction pathways that

C. W. Lim, W. Baek, S. Lim, and S.-W. Han contributed equally to the paper.

Nucleotide and amino acid sequences data reported here have been deposited in the GenBank database under accession numbers KC795276 and AHI85726, respectively.

Corresponding author: Sung Chul Lee; E-mail: sclee1972@cau.ac.kr

*The $\boldsymbol{e}$-Xtra logo stands for "electronic extra" and indicates that four supplementary figures and one supplementary table are published online.

(c) 2015 The American Phytopathological Society may involve efflux of ions, generation of reactive oxygen species, and induction of defense-related genes, ultimately leading to resistance to pathogens. Although defense mechanisms in response to plant-microbe interactions have been widely studied, the mechanisms underlying the functional modifications caused by pathogen infection are unclear. Plants have evolved diverse defense mechanisms to overcome attacks from pathogens. One of these defense mechanisms is to generate defense-related hormones and antimicrobial proteins by increased expression of defense-related genes (Blée 2002; Chen et al. 2009; Lee et al. 2008). The plant defense response to pathogens is regulated by multiple signal transduction pathways, with significant overlap between gene expression patterns induced against different pathogens (Jones and Dangl 2006; Lee et al. 2006b, 2008).

In the defense-activated signal transduction pathways, genes are regulated by the interaction of cis-acting elements in their promoters with multiple transcriptionally or posttranslationally activated transcription factors (Llorca et al. 2014; Thatcher et al. 2012). Transcription factors have specific characteristics, such as nuclear localization, DNA-binding to promoter regions, transcriptional activation or repression, and interaction with other transcription factors or cofactors, which are necessary to regulate target gene expression (Eulgem et al. 2000; Jakoby et al. 2002; Llorca et al. 2014). These transcription factors are then involved in adaptation to unfavorable environmental conditions, such as pathogen infection or osmotic stress (Froidure et al. 2010; Fu and Dong 2013; Lee et al. 2006a). Several transcription factor families, each containing a distinct type of $c$ is-acting element, such as WRKY, ERF, RAV, bZIP, or MYB, have been implicated in plant defense responses, mainly because knock-out or overexpressing mutants exhibited resistant or susceptible phenotypes to different stresses (Kesarwani et al. 2007; Lee et al. 2006a; Li et al. 2014; Sohn et al. 2006; Zhu et al. 2014).

bZIP proteins, one of the largest families of transcription factors in plants, are characterized by a basic region, which is necessary for DNA binding, and a leucine zipper motif, which is responsible for dimerization (Corrêa et al. 2008; Pogenberg et al. 2014). bZIP genes have been reported in all eukaryotes, including Saccharomyces cerevisiae, Homo sapiens, and Arabidopsis thaliana (17, 56, and 75 bZIP proteins, respectively). bZIP is required for homo- or heterodimerization of the protein prior to binding to the promoter region of genes (Baloglu et al. 2014; Corrêa et al. 2008; Llorca et al. 2014; Wang et al. 2011). The potential interactions of each bZIP factor in relation to homo- or heterodimerization are determined by the charge distribution and amino acid position of the zipper region (Llorca 
et al. 2014; Pogenberg et al. 2014). Genetic and molecular studies of bZIP factors in plants have shown that they regulate diverse biological processes, such as seed maturation, senescence, and defense responses to biotic and abiotic stresses (Alves et al. 2013; Jakoby et al. 2002; Zhang et al. 2003). The involvement of bZIP factors in the plant defense response to pathogens is well established (Blanco and Judelson 2005; Lee et al. 2006a; Pitzschke et al. 2009). A common bZIP protein that is linked to defense responses is comprised of the TGA protein, which binds to the activation sequence- 1 (as-1) element found in pathogenesis-related (PR) genes, including the systemice acquired resistance (SAR) marker gene PRl (Després et al. 2003; $\mathrm{Fu}$ and Dong 2013; Kim and Delaney 2002; Pitzschke et al. 2009; Zhang et al. 2003).

In a previous study, we isolated and characterized bZIP transcription factor $C A b Z I P 1$ (Lee et al. 2006a). We reported that transcripts of $C a b Z I P 1$ were accumulated in pepper leaf tissues infected by bacterial pathogens and that CAbZIP1 was involved in plant development. Furthermore, overexpression of CAbZIP1 in Arabidopsis enhanced resistance to pathogens and the plants exhibited increased tolerance to abiotic stresses, including drought and high salinity. In this study, we identified and functionally characterized a pepper bZIP transcription factor, $C a b Z I P 2$, which was isolated from a pepper leaf cDNA library using pathogen-inducible cDNA as a probe. To investigate the functional role of the CabZIP2 protein as a transacting factor, GAL4 fusion proteins were analyzed in yeast. Using CabZIP2-silenced pepper plants and CabZIP2 overexpressing transgenic Arabidopsis plants, we evaluated the in vivo functions of $C a b Z I P 2$ based on the expression profile of the pepper CabZIP2 gene in response to bacterial pathogens and abiotic elicitors. Our data suggest that CabZIP2 is involved in the expression of defense-related genes during pathogen infection. These findings suggest that the CabZIP2 transcription factor acts as a positive regulator of defense signaling.

\section{RESULTS AND DISCUSSION}

\section{Isolation and sequence analysis of the CabZIP2 gene.}

To isolate novel pathogen-induced pepper genes, we performed differential hybridization screening of a cDNA library constructed using leaves infected with an avirulent strain, Bv5$4 \mathrm{a}$, of $X$. campestris pv. vesicatoria, and the putative pepper pathogen-induced candidate gene was isolated (Jung and Hwang 2000). We designated this gene CabZIP2 (Capsicum annuиm bZIP transcription factor 2), based on sequencing and amino acid alignment (Fig. 1). The CabZIP2 clone consists of $1,243 \mathrm{bp}$, and the deduced amino acid sequence encodes a protein of 344 amino acids containing a nuclear localization signal (Fig. 1). Sequence analysis revealed that CabZIP2 contains an $\mathrm{N}$-terminal region that includes an acidic domain, a bZIP region, and a C-terminal region. The bZIP domain, composed of 59 amino acids and present in the central region of CabZIP2, was extensively characterized in various plant proteins. Amino acid sequence alignments and comparison of CabZIP2 with six bZIP transcription factor members in different plant species revealed that these bZIP proteins have similar leucine zipper structural signatures (Fig. 1). The predicted CabZIP2 (accession number AHI85726) shares $86.3 \%$ identity with the potato VIP1-like protein (accession number XP_006362824), 85.2\% identity with the tobacco RSG protein (accession number BAA97100) and tomato VIP1-like protein (accession number XP_004237777), $60.2 \%$ identity with the pepper bZIP transcriptional activator (accession number ACB30357), 53.53\% identity with the Arabidopsis VIP1 protein (accession number NP_001237194), and $52.3 \%$ identity with the soybean bZIP131 protein (accession number NP_001237194). These sequence differences were reflected in a phylogenetic tree, and CabZIP2 was grouped in the same clade with six VIP1-like proteins containing CabZIP (accession number ACB30357; 73\% identity and 85\% similarity) (Supplementary Fig. S1). In particular, CabZIP1 (accession number AAX20030; Lee et al. 2006a), isolated in our previous study, shares $19.4 \%$ identity with CabZIP2 and was located in a different clade, indicating CabZIP2 is a novel gene isolated from pepper.

\section{Subcellular localization of CabZIP2.}

To determine the subcellular localization of the CabZIP2 protein, we fused the green fluorescent protein (GFP) sequence to $C a b Z I P 2$ cDNA under the control of the $35 \mathrm{~S}$ promoter (Fig. 2). As shown in Figure 2, expression of the 35S:CabZIP2-GFP fusion protein in $N$. benthamiana epidermal cells generated GFP signals in the nucleus and cytoplasm, coincident with 4',6diamidino-2-phenylindole staining, indicating that CabZIP2 is a cytosolic- as well as a nuclear-localized protein. Figure 1 shows that CabZIP2 has high homology to VIP1-like proteins in other plant species. Djamei et al. (2007) suggested that VIR1 is relocated from the cytoplasm to the nucleus by flg22, a bacterial PAMP. To determine whether the localization of CabZIP2 was affected by bacterial PAMP, $N$. benthamiana leaves expressing 35S:CabZIP2-GFP were treated with flg22, while localization of CabZIP2 was not changed (Supplementary Fig. S2). A fluorescent signal was detected in the nucleus and cytoplasm, indicating that PAMP did not change CabZIP2 localization. Although the localization of CabZIP2 protein was not affected by this PAMP, it is possible that the levels of CabZIP2 proteins present are sufficient for function in the nucleus.

\section{Transactivation assay of CabZIP2 in yeast.}

Transcription factors have an activation domain that is enriched in either proline, glutamine, or acidic amino acids. However, not all activation domains fit into these categories (Cantin et al. 2003; Jakoby et al. 2002; Schwechheimer et al. 1998a and b). To determine whether the CabZIP2 protein could function as a transactivator in yeast, a series of deletion constructs of the CabZIP2 gene were fused to the vector for the yeast GAL4 DNA binding domain (GAL4DB) to be expressed under the control of the Saccharomyces cerevisiae ADH1 promoter (Fig. 3A). The fusion proteins were expressed in Saccharomyces cerevisiae AH109, which carries integrated copies of nutritional reporter genes (ADEl and $H I S 3$ ) under the control of GAL4-responsive promoters (bait::full length [BF]: 1 to 344, bait::N-terminal domain [BN]: 1 to 130, bait:: bZIP domain [BZ]: 131 to 260 , and bait::C-terminal domain [BC]: 261 to 334). Transcription activation of these constructs was confirmed by growing yeast cells in selection medium (SD [-Ade, -His, -Leu, -Trp]). CabZIP2 contains an acidic domain in its N-terminal region, similar to that in other bZIP transcription factors. Acidic transactivation domains have universal functions in eukaryotes (Hahn 1993; Schwechheimer et al. 1998b). Yeast cells carrying the constructs containing $\mathrm{BF}$ or $\mathrm{BN}$ grew well on the selection medium (Fig. 3A), indicating that expression of the ADE1 and HIS3 reporter genes was activated by the N-terminal domain of CabZIP2. The N-terminal region including the acidic domain of CabZIP2 functions as a transactivation domain in yeast. In contrast, the constructs expressing the bZIP region (BZ: 131 to 260) and the C-terminal region (amino acids 261 to 334) did not cause yeast growth in the selection medium. These results suggest that the N-terminal region including the acidic domain of CabZIP2 is responsible for its ability as a potential transcriptional activator in yeast.

Several studies have reported that bZIP transcription factors have dimerization preferences from different species, including Homo sapiens, Drosophila melanogaster, Saccharomyces cerevisiae, and Arabidopsis thaliana (Deppmann et al. 2006). Unlike most other bZIP transcription factors, subfamily I members are predicted to form homodimers rather than heterodimers. Since CabZIP2 belongs 
to subfamily I of bZIP proteins, its homodimerization was verified by a bimolecular fluorescence complementation (BiFC) assay. As shown in Figure 3B, An Agrobacterium-mediated transient expression assay in $N$. benthamiana leaves was performed to confirm the homodimerization of CabZIP2. Coexpression of CabZIP2: SPYNE with CabZIP2:SPYCE resulted in yellow fluorescence only in the cytoplasm and nucleus. This result indicates that CabZIP2 presumably functions as a homodimer. Protein dimerization of bZIP pairs in this family of transcription factors is mediated by the leucine zipper motif, which is required for the recognition of cis-acting elements (Deppmann et al. 2006; Llorca et al. 2014). In contrast, some bZIP transcription factors lose their DNA-binding activity when they form heterodimers with certain partners (Kang et al. 2010).

Induction of CabZIP2 gene expression in pepper leaves by bacterial pathogen infection and abiotic elicitors.

Reverse transcription-polymerase chain reaction (RT-PCR) analysis was performed to examine whether CabZIP2 is constitutively expressed in pepper tissues, such as leaf, stem, root, and flower (Supplementary Fig. S3). CabZIP2 was found to be constitutively expressed in all these tissues and the expression levels of CabZIP2 were relatively high especially in the stem and root tissues. To determine whether CabZIP2 is induced by compatible and incompatible interactions of pepper with $X$. campestris pv. vesicatoria, the expression levels of CabZIP2 were analyzed in pepper leaves inoculated with virulent and avirulent strains of $X$. campestris pv. vesicatoria (Fig. 4A). Pepper leaves inoculated with virulent strain Ds1 did not show any visible symptoms $24 \mathrm{~h}$ after inoculation, whereas chlorotic and necrotic symptoms were visible 6 days after inoculation. In contrast, pepper leaves infected with avirulent strain Bv5-4a exhibited a hypersensitive response $18 \mathrm{~h}$ after inoculation (Lee and Hwang 1996). This different disease response results in the differential induction of CabZIP2. In the compatible interaction, $C a b Z I P 2$ transcripts were first detected $2 \mathrm{~h}$ after inoculation, with maximal levels at $6 \mathrm{~h}$. In the incompatible interaction, the CabZIP2 transcripts were more rapidly and strongly accumulated

$\mathrm{AD}$

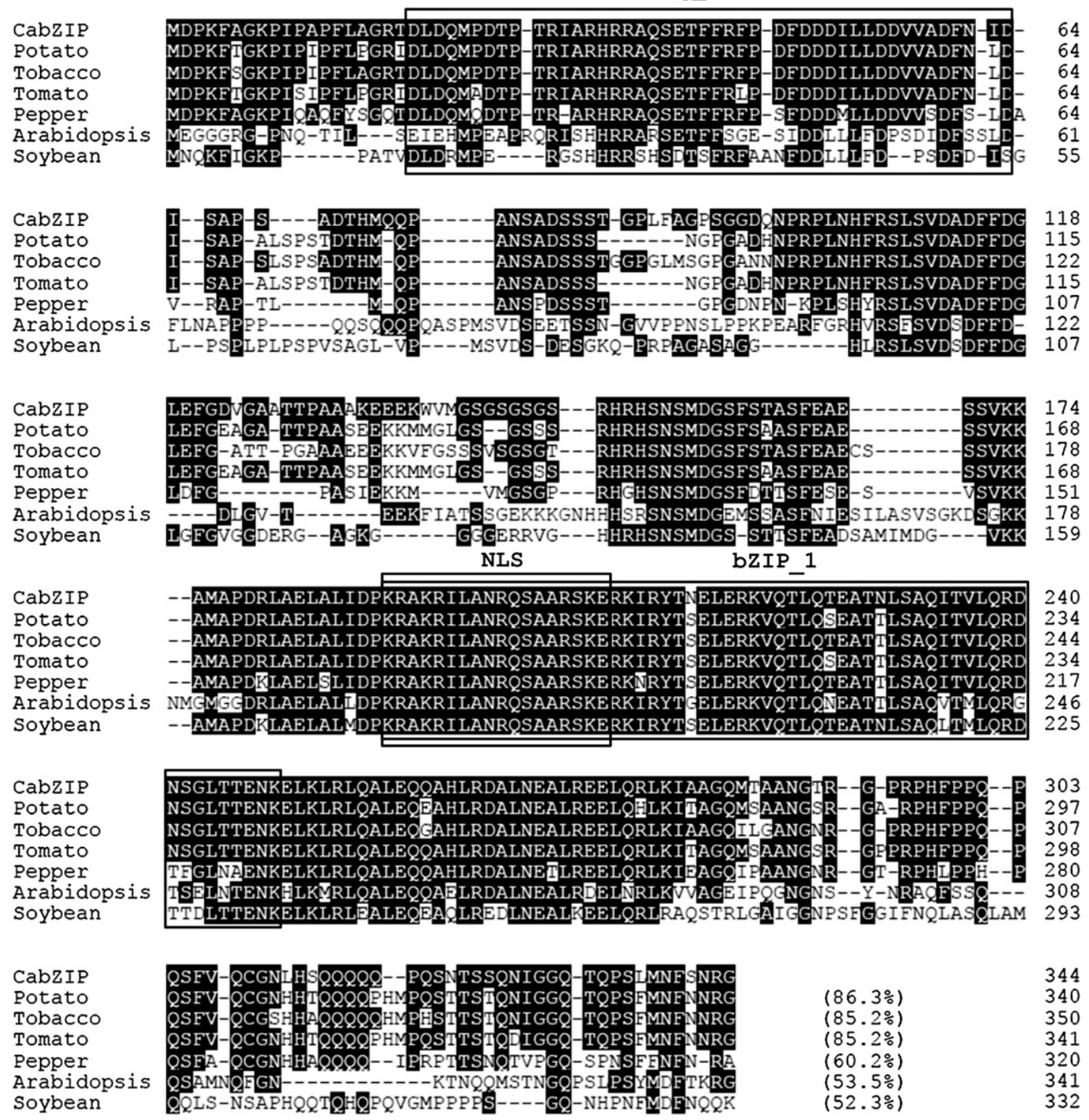

Fig. 1. Alignment of amino acid sequences of pepper CabZIP2 cDNA. Comparison of the deduced amino acid sequences of the pepper CabZIP2 protein with the bZIP transcription factor from Solanum tuberosum (accession number XP_006362824), Nicotiana tabacum (BAA97100), Solanum lycopersicum (XP_004237777), Capsicum annuum (ACB30357), Arabidopsis thaliana (NP_001237194), and Glycine max (NP_001237194) proteins. White letters on black indicate identical amino acid residues. The acidic domain (AD), nuclear localization signal (NLS), and bZIP domain are represented by black boxs. Gaps introduced to maximize the alignment of homologous regions are marked by dashes. 
compared with that observed during the compatible interaction. The CabZIP2 gene was detectable $0.5 \mathrm{~h}$ after inoculation, followed by a slight and gradual reduction of expression. The defense signals generated during the earlier stages of the plant-

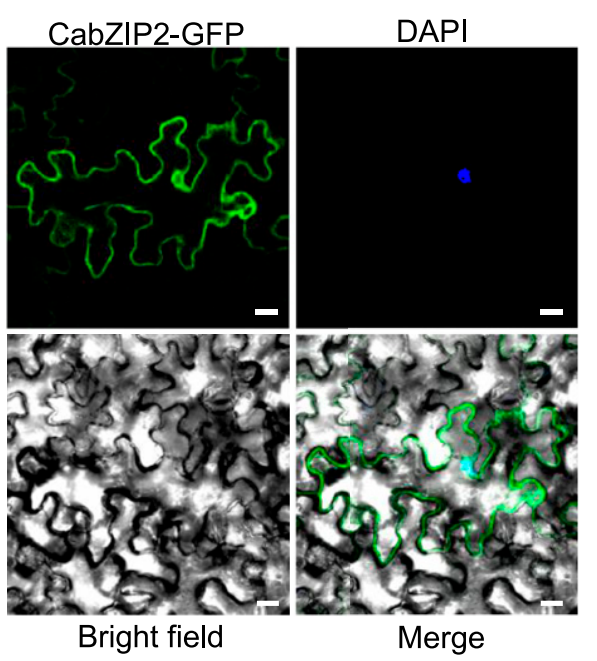

Fig. 2. Subcellular localization of the CabZIP2 protein using transient expression of the 35S:CabZIP2-GFP construct in Nicotiana benthamiana. Transient expression of 35S:CabZIP2-GFP in N. benthamiana was examined $48 \mathrm{~h}$ after transformation, under a Zeiss LSM 700 confocal laserscanning microscope. 4',6-Diamidino-2-phenylindole staining indicates the positions of nuclei in cells. The scale bar represents $101 \mathrm{~m}$. microbe interactions are required for an effective defense response against the pathogen and may be integrated into one of the terminal pathways that leads to the transcriptional activation of PR genes (Lippok et al. 2007; Boyle and Després 2010). In the compatible and incompatible interactions, strong expression of CabZIP2 occurred at 0.5 and $2 \mathrm{~h}$ after inoculation, respectively. In our earlier studies, transcription of pepper PR genes, including $C A B P R 1$ and $C a A M P 1$, was induced by bacterial pathogens (Hong et al. 2005; Lee et al. 2008); therefore, we compared expression patterns of these genes with CabZIP2 (Supplementary Fig. S4). Expressions of CaBPRI and CaAMPl occurred after the induction of CabZIP2. Furthermore, CabZIP2 transcripts were induced more rapidly and strongly during incompatible interactions as compared with that in compatible interactions, which suggests that the CabZIP2 gene may be involved in the defense response.

The plant hormones salicylic acid (SA), ethylene (ET), and methyl jasmonate (MeJA) are already known as defense-related hormones that may accumulate during pathogen infection and are involved in the signal transduction pathway (Alazem and Lin 2014). To assess the effect of these hormones on CabZIP2 expression, we applied SA, ET, or MeJA to pepper leaves. SA has been reported to be a key component in the signal transduction pathway leading to plant resistance to biotrophic pathogens (Lazebnik et al. 2014). Treatment with SA strongly upregulated CabZIP2 within 0.5 to $12 \mathrm{~h}$ after treatment (Fig. 5B). Following ET treatment, CabZIP2 transcripts were first detected after $0.5 \mathrm{~h}$ and increased rapidly between 6 and $12 \mathrm{~h}$ after treatment (Fig. 5C). In MeJA-treated pepper leaves, CabZIP2 transcripts started to accumulate $0.5 \mathrm{~h}$ after treatment

A

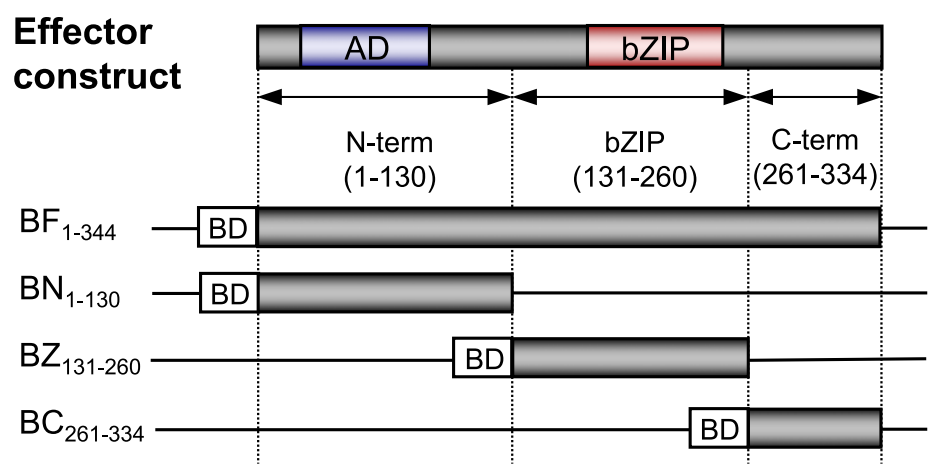

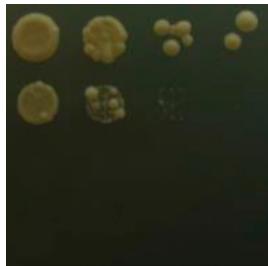

SD-AHLW

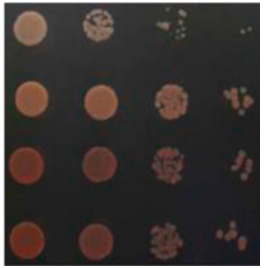

SD-LW

\section{Reporter

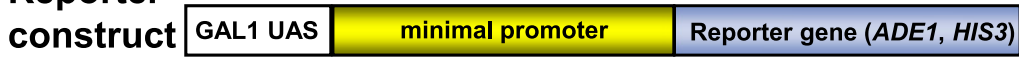

B
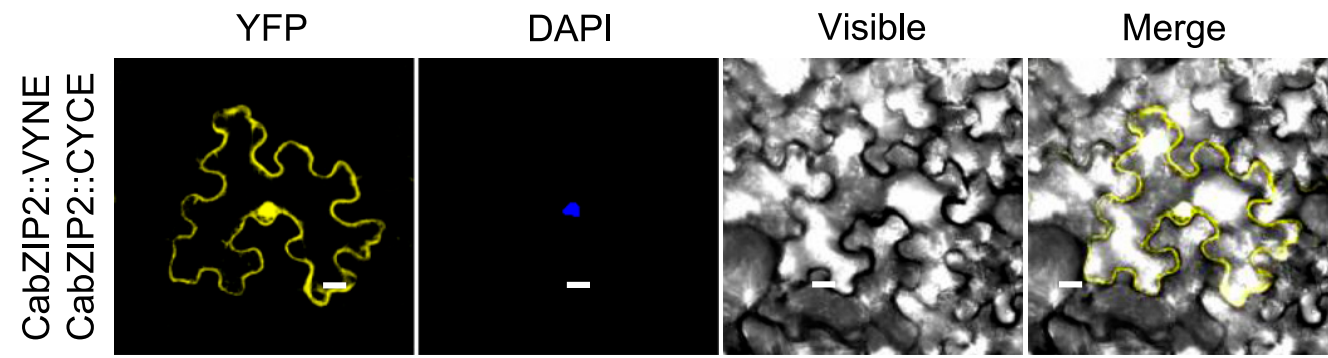

Fig. 3. Functional analysis of the CabZIP2 protein. A, Transactivation of GAL4-responsive transcription by the full-length and truncated forms of the CabZIP2 gene, using a GAL4 yeast system. Derivatives of yeast strain AH 109, harboring plasmids that encode GAL4-CabZIP2, were grown on SD medium (-Leu, -Trp; right panel) lacking adenine and histidine (-Ade, $-\mathrm{His},-$ Leu, $-\mathrm{Trp}$; left panel). Plates were incubated at $28^{\circ} \mathrm{C}$ for 5 days. BD = binding domain; $\mathrm{AD}=$ activation domain. B, Analysis of homodimerization of CabZIP2 proteins by the bimolecular fluorescence complementation assay. CabZIP2-35SSPYNE(R) 173 coexpressed with CaBZIP2-35S-SPYCE(M) in the leaves of Nicotiana benthamiana by agroinfiltration. The fluorescence indicates interaction between CabZIP2 proteins. Images were obtained from the yellow fluorescent protein channel, bright field, or a merged picture of the two. White bar $=101 \mathrm{~m}$. 
and reached peak levels at 2 to $12 \mathrm{~h}$ (Fig. 5D). These defenserelated hormones are known to trigger disease resistance in plants (Adie et al. 2007; Clarke et al. 2000; Ferrari et al. 2003). The expression of CabZIP2 was induced more rapidly and extensively in the incompatible interactions and following treatment with defense hormones, which suggests that the CabZIP2 gene may be involved in the defense response.

\section{Enhanced susceptibility of CabZIP2-silenced} pepper plants to $X$. campestris pv. vesicatoria infection.

Since the experiments described above show that CabZIP2 gene expression in pepper leaves is activated by $X$. campestris pv. vesicatoria infection, and abiotic elicitor treatment to trigger defense signaling (Fig. 4), we examined the role of CabZIP2 in the defense response of pepper plants to bacterial pathogens, using the virus-induced gene silencing (VIGS) technique. The generation of stable transgenic pepper plants by overexpressing or knock-out genes of interest is very difficult, and VIGS analysis provided a fast and effective means to knock-down phenotypes and allowed us to elucidate the function of interesting genes in pepper plants (Lee et al. 2010; Lim and Lee 2014). Pepper seedlings were inoculated with recombinant Tobacco rattle virus (TRV) silencing constructs. Phenotypic differences were not observed in plants containing an empty-vector control (TRV:00) or in CabZIP2-silenced (TRV:CabZIP2) pepper plants during growth and development (data not shown). The efficiency of VIGS was analyzed by quantitative (q)RT-PCR using CabZIP2 empty-vector control and CabZIP2-silenced pepper leaves $12 \mathrm{~h}$ after inoculation with virulent (Ds1) and avirulent (Bv5-4a) strains of $X$. campestris pv. vesicatoria (Fig. 5A). CabZIP2 expression was compromised in CabZIP2-silenced pepper leaves with or without inoculation of $X$. campestris pv. vesicatoria. Since CabZIP2 functions as a transcriptional activator (Fig. 3) and the VIP1 transcription factor regulates the expression of PR1 (Djamei et al. 2007), we examined by RTPCR whether CabZIP2 silencing affects the induction of defense-related genes (Fig. 5A). The expression levels of CaBPRl (Hong et al. 2005) and CaAMP1 (Lee et al. 2008) were remarkably reduced in $C a b Z I P 2$-silenced pepper leaves as compared with empty-vector control plants.

To confirm whether the expression of defense-related genes, including CabZIP2, reflected bacterial proliferation within the plants, bacterial growth was measured in empty-vector control and $C a b Z I P 2$-silenced pepper at 0,3 , or 5 days postinoculation (dpi) (Fig. 5B and C). In the compatible interaction, silencing of CabZIP2 not only compromised the expression of some defenserelated genes, it also increased susceptibility of plants to infection by the virulent strain of $X$. campestris pv. vesicatoria (Fig. 5B). CabZIP2-silenced plants exhibited significantly higher bacterial proliferation than control plants at 3 or $5 \mathrm{dpi}$. Consistent with the induction of CabZIP2 expression by infection of $X$. campestris pv. vesicatoria and treatment of abiotic elicitors, these findings strongly support the notion that CabZIP2 may be required for the resistance of pepper plants to virulent bacterial pathogens. In the incompatible interaction, bacterial proliferation was not distinguished in CabZIP2-silenced pepper leaves compared with empty-vector control pepper leaves (Fig. 5C). Although CabZIP2 expression was rapidly and strongly induced in pepper leaves by avirulent $X$. campestris pv. vesicatoria infection, CabZIP2 silencing did not significantly alter the susceptibility of pepper plants to avirulent $X$. campestris pv. vesicatoria infection, indicating that $C a b Z I P 2$ plays a key role in basal resistance rather than in resistance gene-mediated resistance. Together, these results indicate that CabZIP2 affects the expression of other

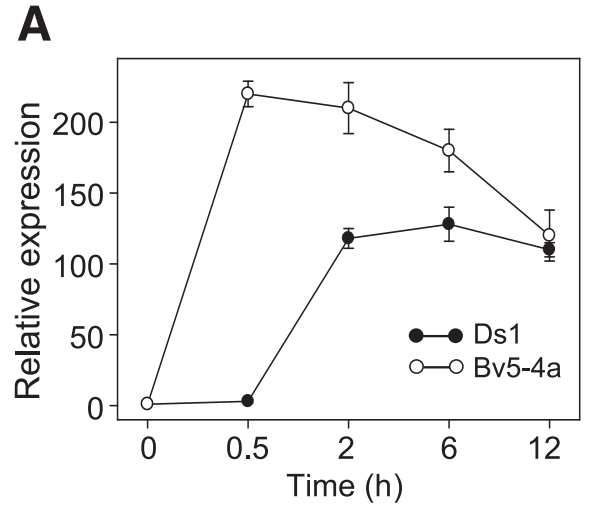

B
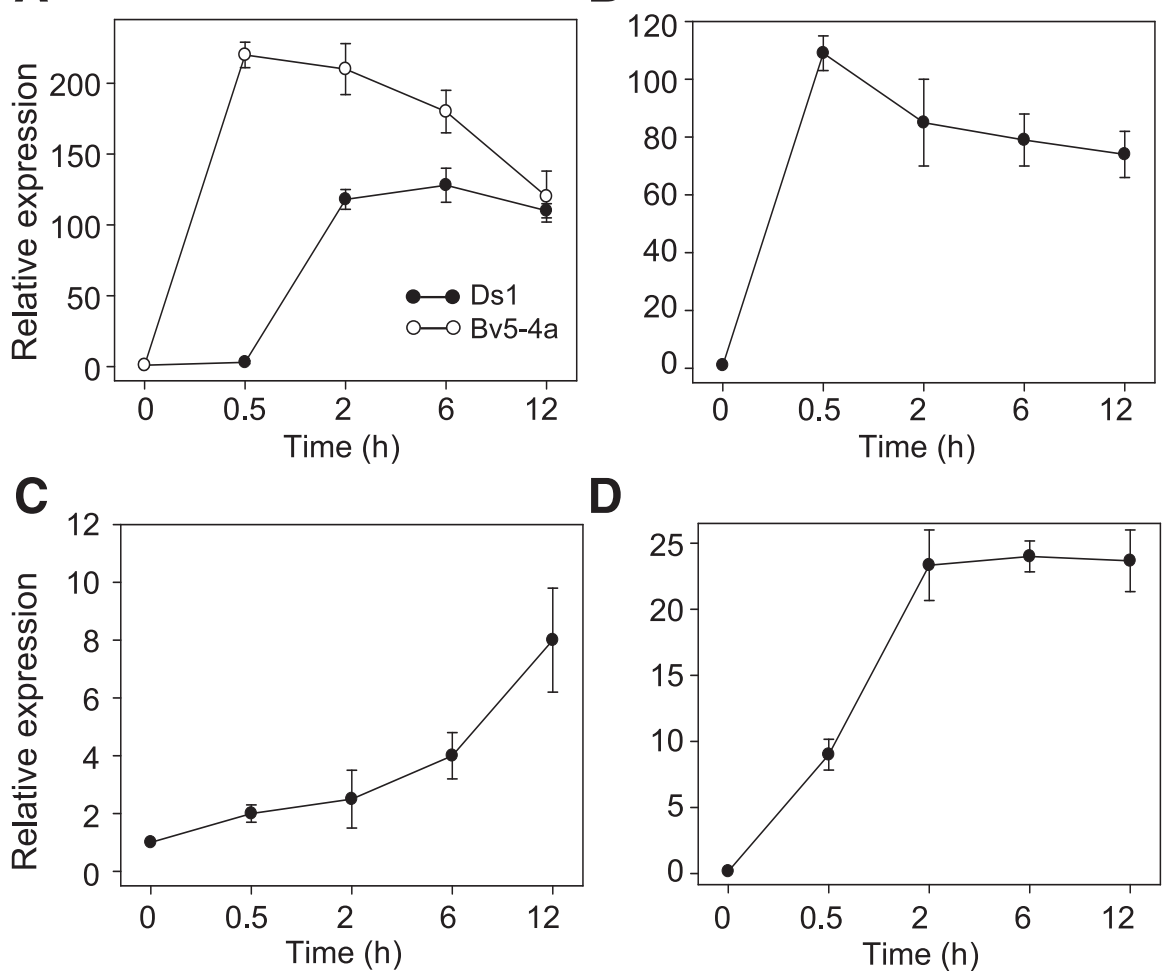

D

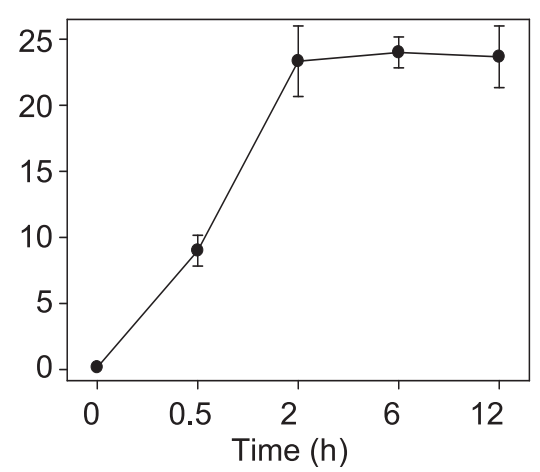

Fig. 4. Relative expression of the CabZIP2 gene in pepper plants. A, Expression of CabZIP2 in pepper leaves at various time points after inoculation with the virulent strain Ds1 and the avirulent strain Bv5-4a of Xanthomonas campestris pv. vesicatoria at the four-leaf stage. B to D, Expression of CabZIP2 in pepper leaves at various time points after treatment with salicylic acid (B), ethylene (C), and methyl jasmonate (D). Relative expression levels ( $\triangle \triangle \mathrm{CT}$ ) of the $C a b Z I P 2$ gene were normalized to the geometric mean of $18 \mathrm{~s} r \mathrm{RNA}$, Actin1, and EF1 $\alpha$ expression as internal control genes and were compared with each value for mocktreated leaves. Data are expressed as the mean \pm standard error from three independent experiments. 
defense-related genes and plays a crucial role in the defense response of pepper plants to bacterial pathogens.

\section{Enhanced resistance of CabZIP2-OX (overexpressing) transgenic plants to bacterial pathogen.}

To further investigate the effect of CabZIP2 in the defense response in vivo, we generated CabZIP2 Arabidopsis transgenic plants that overexpress the entire precursor under the control of a strong constitutive $35 \mathrm{~S}$ promoter (Fig. 6). To verify CabZIP2-OX transgene expression in independent homozygous $\mathrm{T}_{3}$ lines, we performed RT-PCR analysis using genomic mRNA from the wild-type plants and from the CabZIP2-OX plants. PCR products were not detected for the wild-type plants, while the CabZIP2 gene was amplified from three CabZIP2OX plants, which were used for further phenotypic analysis (Fig. 6A). The phenotype of CabZIP2-OX plants was indistinguishable from wild-type plants under normal growth conditions (data not shown). To examine whether overexpression of CabZIP2 was able to enhance disease resistance, we inoculated both the wild-type and CabZIP2-OX plants with $P$. syringae pv. tomato DC3000, which causes symptoms similar to bacterial speck disease of tomato and Arabidopsis (Whalen et al. 1991). Seven days after inoculation, wild-type plants exhibited typical chlorotic symptoms on inoculated leaves, whereas symptoms were not visible on any leaves of the CabZIP2-OX lines (Fig. 6B). To confirm that these macroscopic disease symptoms reflect bacterial proliferation in the plants, bacterial growth in the leaf tissues was examined at 0,1 , 3 , and 5 dpi (Fig. 6C). The bacterial growth was not any different in wild-type and CabZIP2-OX plants at 0 or $1 \mathrm{dpi}$. The CabZIP2-OX plants showed a 15 - to 30 -fold reduction in bacterial growth at $3 \mathrm{dpi}$. At $5 \mathrm{dpi}$, the bacterial population in wild-type plants was only slightly reduced and the CabZIP2OX plants showed 18- to 20-fold less bacterial growth of $P$. syringae pv. tomato DC 3000 , as compared with that in the wild-type plants. These results indicate that overexpression of CabZIP2 confers enhanced resistance to bacterial pathogens. Enhanced resistance of the CabZIP2 transgenic lines to the virulent bacterial pathogen $P$. syringae pv. tomato DC3000 presumably arises from the overexpression of the pepper CabZIP2 gene. In our previous study, CAbZIP1-OX plants also exhibited a phenotype that was resistant to $P$. syringae pv. tomato DC3000. However, CAbZIP1 is a different bZIP transcription factor from CabZIP2 (Lee et al. 2006a).

To investigate whether this enhanced resistance to the bacterial pathogen in CabZIP2-OX plants is related to SAdependent PR gene expression, we compared the expression levels of PRI and NPR1 in wild-type and CabZIP2-OX plants (Fig. 7). The transcripts of these genes were more-accumulated in leaves of CabZIP2-OX than in leaves of wild-type plants. However, the expression levels of PDF1.2 and LOX genes, which are related to JA, were not significantly different in wildtype and CabZIP2-OX plants. These results indicate that a SAdependent defense mechanism may contribute to CabZIP2-OX enhanced disease resistance to bacterial pathogen infection.

In conclusion, we have shown that the CabZIP2 protein localizes to both the cytoplasm and the nucleus, in which it acts as a transcriptional activator. Silencing of CabZIP2 led to an
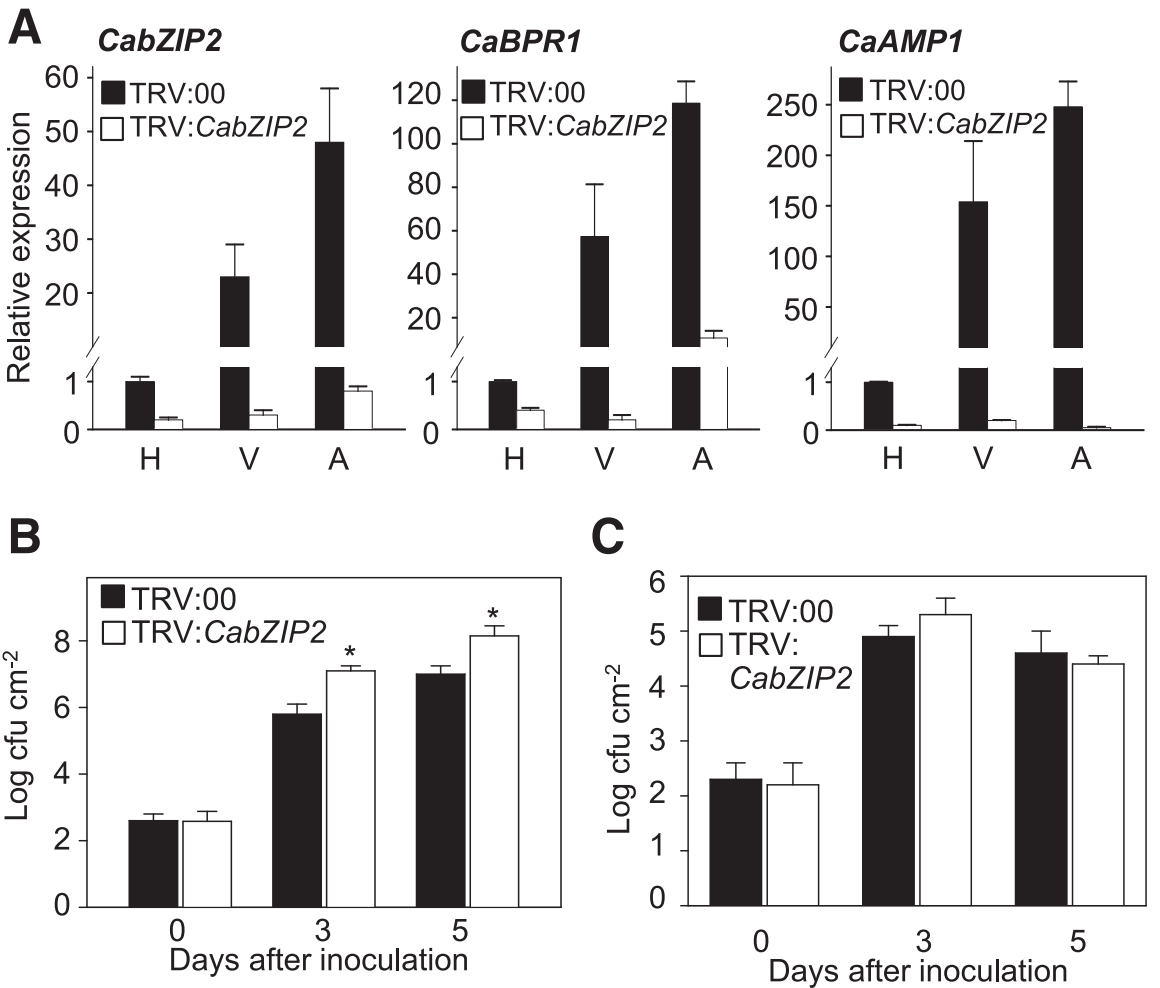

Fig. 5. Enhanced susceptibility of CabZIP2 gene-silenced pepper plants to Xanthomonas campestris pv. vesicatoria infection. A, Expression of CabZIP2 and pepper defense-related genes in empty-vector control (TRV:00) and CabZIP2 gene-silenced (TRV: CabZIP2) pepper plants 12 h after inoculation with the $X$. campestris pv. vesicatoria virulent strain Ds1 and the avirulent strain Bv5-4a. $18 S$ rRNA served as a control. $\mathrm{H}=$ untreated healthy leaves, $\mathrm{V}=$ virulent strain Ds1, A = avirulent strain Bv5-4a, CaAMP1 = pepper antimicrobial protein 1 , and $C a B P R 1=$ pepper basic PR1. Reative expression levels $(\triangle \triangle \mathrm{CT})$ of the CabZIP2 gene were normalized to the geometric mean of $18 \mathrm{~s} r R A$, Actin 1, and EF1 $\alpha$ expression as internal control genes and were compared with the values for nontreated leaves. Data are expressed as the mean \pm standard error from three independent experiments. B and $\mathbf{C}$, Bacterial growth in empty-vector control and CabZIP2 gene-silenced pepper leaves at 0,3, and 5 days after inoculation with the X. campestris pv. vesicatoria virulent strain Ds 1 (B) and avirulent strain Bv5-4a $\left(10^{4} \mathrm{CFU} \mathrm{ml}{ }^{-1}\right)(\mathrm{C})$. Experiments were repeated three times with similar results. Data are expressed as the mean \pm standard deviation from three independent experiments. Asterisks indicate significant difference as determined by Student's $t$ test $(P<0.05)$. 
enhanced susceptibility of pepper plants to the bacterial pathogen, whereas ectopic expression of the CabZIP2 transgenic Arabidopsis plants induces enhanced resistance to $P$. syringae pv. tomato DC3000. Together, these results suggest that CabZIP2 may function as a transcriptional activator of the defense response against bacterial pathogens in pepper plants. However, the mechanisms involved in the activation of the defense response by CabZIP2 remain unclear. Furthermore, stable transgenic pepper plants, in which CabZIP2 is knockedout, are required to better understand the function of this gene.

\section{MATERIALS AND METHODS}

\section{Plant material and growth conditions.}

Pepper (Capsicum annuum L. cv. Nockwang), Arabidopsis thaliana (ecotype Col-0), and Nicotiana benthamiana plants were grown at a 9:1:1 ratio of peat moss, perlite, and vermiculite under fluorescent light (130 $1 \mathrm{~mol}$ photons $\mathrm{m}^{-2} \mathrm{~s}^{-1}$ ) at $24^{\circ} \mathrm{C}$ with $60 \%$ humidity and a 16-h light and 8-h dark cycle. Arabidopsis thaliana seeds were surface-sterilized with $70 \%$ ethanol for $1 \mathrm{~min}$ prior to in vitro culture. After the seeds were treated with $2 \%$ sodium hydroxide for $10 \mathrm{~min}$, they were
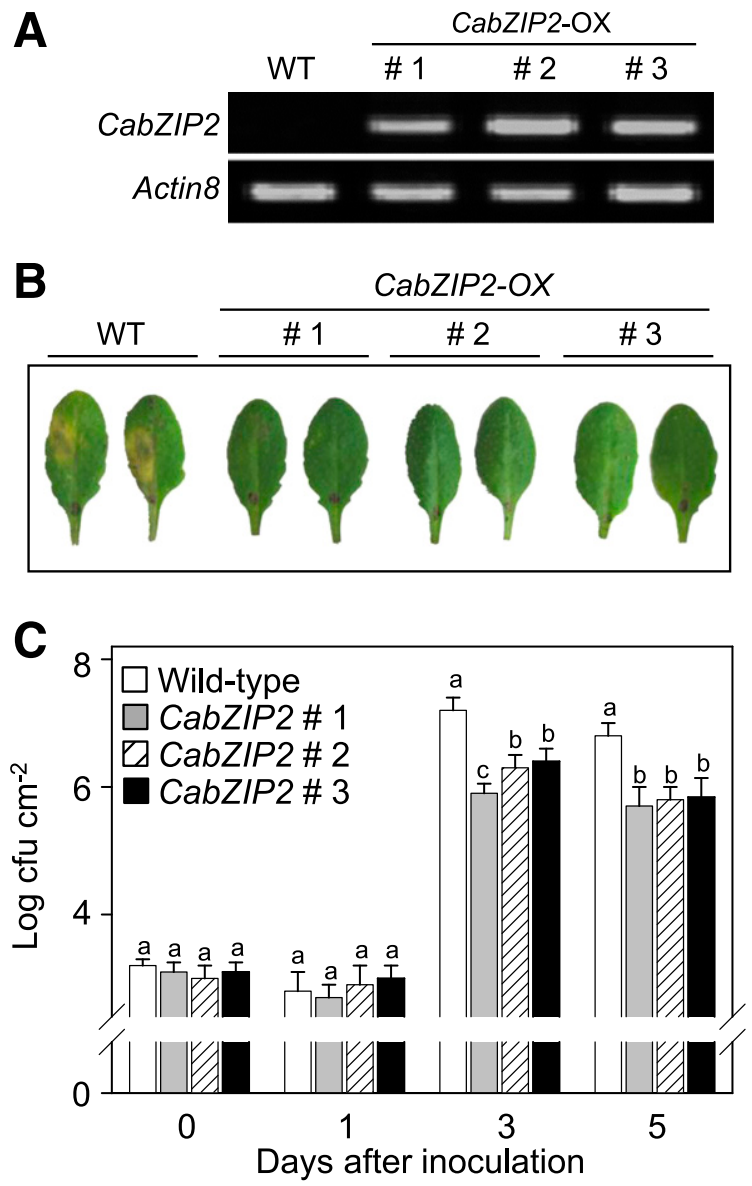

Fig. 6. Enhanced resistance of CabZIP2-overexpressing (OX) transgenic Arabidopsis plants to Pseudomonas syringae pv. tomato DC3000 infection. A, Reverse transcription-polymerase chain reaction analysis of CabZIP2 expression in wild type and the CabZIP2-OX transgenic lines. Actin8 was used as internal control gene. B, Disease symptoms developed on the leaves of wild-type or transgenic plants 7 days after infiltration with $P$. syringae pv. tomato DC3000 $\left(10^{5} \mathrm{CFU} \mathrm{ml}^{-1}\right)$. C, Bacterial growth in the leaves of wild-type and transgenic plants at different time points after infiltration with $P$. syringae pv. tomato DC3000 $\left(10^{5} \mathrm{CFU} \mathrm{ml}^{-1}\right)$. Values are the mean \pm standard deviation from three independent experiments. Statistical analysis was performed with analysis of variance test $(P<0.05)$ and different letters indicate significant differences. washed 10 times in sterile distilled water and were sown on Murashige and Skoog (MS) (Murashige and Skoog 1962) agar supplemented with $1 \%$ sucrose for 2 weeks at $24^{\circ} \mathrm{C}$ in a growth chamber exposed to a 16-h light and 8-h dark cycle.

\section{Pathogen inoculation.}

Strains of Xanthomonas campestris pv. vesicatoria that are virulent (Ds1) and avirulent (Bv5-4a) to pepper were used in this study. To prepare bacterial suspensions for inoculation of pepper leaves, bacteria were cultured for $16 \mathrm{~h}$ in yeast-nutrient broth $(5 \mathrm{~g}$ of yeast extract, $8 \mathrm{~g}$ of nutrient broth, and 1 liter of $\mathrm{H}_{2} \mathrm{O}$ ) at $28^{\circ} \mathrm{C}$. Bacterial suspensions were adjusted to a concentration of $10^{8} \mathrm{CFU} \mathrm{ml} \mathrm{m}^{-1}$ with sterile tap water prior to inoculation. Pepper plants at the six-leaf stage were inoculated by infiltrating bacterial suspensions into the abaxial side of fully expanded leaves. The control healthy plants also were mockinoculated by infiltrating sterile tap water into the leaves. The mock-inoculated and bacteria-inoculated pepper plants were incubated in a growth room as described previously (Lee and Hwang 2009).

The virulent pathogen $P$. syringae pv. tomato DC3000 was used in this study. To prepare bacterial suspensions for inoculation on Arabidopsis leaves, bacterial strains were cultured overnight in King's B medium broth at $28^{\circ} \mathrm{C}$. Leaves of 5week-old plants were syringe-inoculated with the bacterial suspension of $P$. syringae pv. tomato DC3000 $\left(1 \times 10^{5} \mathrm{CFU}\right.$ $\mathrm{ml}^{-1}$ ) in $10 \mathrm{mM} \mathrm{MgCl}_{2}$. After inoculation, Arabidopsis plants were maintained under $100 \%$ humidity for $24 \mathrm{~h}$ and were then raised in a growth chamber under the same conditions. The density of the bacterial population was determined by plating serial dilutions on King's B medium supplemented with kanamycin $\left(501 \mathrm{~g} \mathrm{ml}^{-1}\right)$ and rifampicin $\left(501 \mathrm{~g} \mathrm{ml}^{-1}\right)$ at $28^{\circ} \mathrm{C}$. Data are means \pm standard deviations of the $\log \left(\mathrm{CFU} \mathrm{cm}{ }^{-2}\right)$ of three replicates. Bacterial growth in the infected leaves of each plant was determined according to the method of Katagiri et al. (2002).

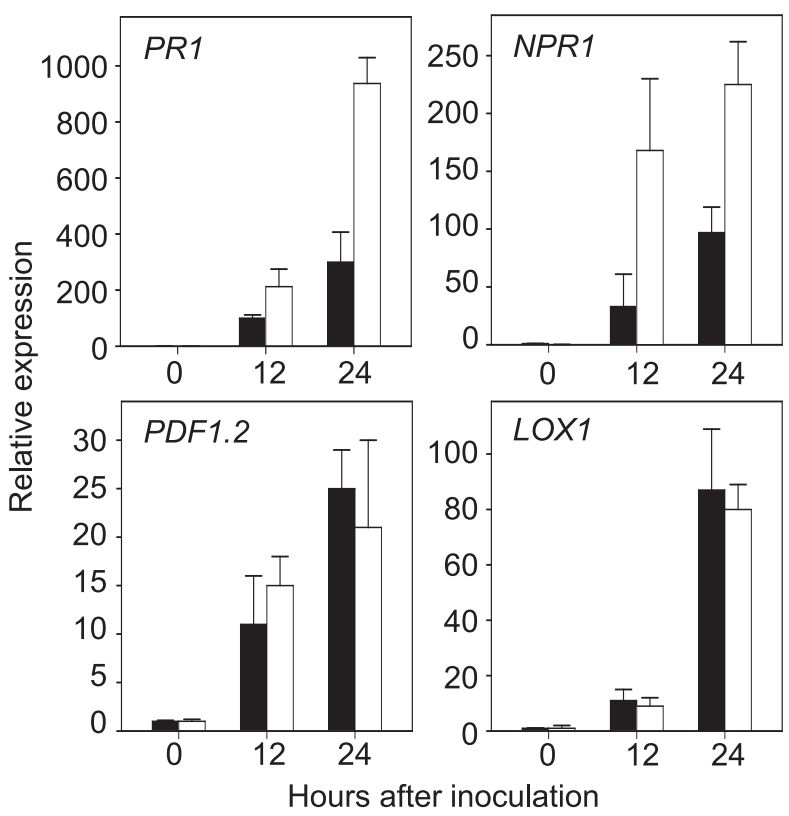

Fig. 7. Relative expression of (pathogenesis related) $P R$ genes in leaves of wild-type and transgenic plants after infiltration with Pseudomonas syringae pv. tomato DC3000 $\left(10^{5}\right.$ cell $\left./ \mathrm{ml}\right)$. Relative expression levels $(\triangle \Delta \mathrm{CT})$ of each gene were normalized to the geometric mean of Actin2, Actin8, and $E F 1 \alpha$ as internal control genes. Values are the mean \pm standard deviation from three independent experiments. Asterisks indicate significant difference as determined by Student's $t$ test $(P<0.05)$. 


\section{Treatments with plant hormones.}

Pepper plants at the six-leaf stage were enclosed in stoppered 500-ml glass bottles, and ethylene was injected to yield a final concentration of 511 per liter. MeJA (100 1M) and SA (5mM) were sprayed onto pepper plants at the six-leaf stage. Pepper plants treated with MeJA were incubated in a vinyl bag. Control plants were sprayed with water. The treated pepper plants were incubated in a growth room at $27^{\circ} \mathrm{C} \pm 1^{\circ} \mathrm{C}$ under approximately $801 \mathrm{~mol}$ photons $\mathrm{m}^{-2} \mathrm{~s}^{-1}$ (white fluorescent lamps) for $16 \mathrm{~h}$ a day.

RNA isolation, RT-PCR, and real-time qRT-PCR analysis.

Total RNA was isolated from pepper leaf tissues infected with bacterial pathogen or abiotic plant hormones, using an RNeasy mini kit (Qiagen, Valencia, CA, U.S.A.), according to the manufacturer's instructions. To remove genomic DNA, all RNA samples were treated with RNA-free DNase. After quantification using a spectrophotometer, $11 \mathrm{~g}$ of total RNA was used to synthesize cDNA using a Transcript First Strand cDNA synthesis kit (Roche, Indianapolis, IN, U.S.A.), according to the manufacturer's instructions. In parallel, cDNAs were synthesized without reverse transcription and were subjected to qRT-PCR to rule out the possibility of contamination by genomic DNA in the cDNA samples. The synthesized cDNA was amplified in a CFX96 Touch Real-Time PCR detection system (Bio-Rad, Hercules, CA, U.S.A.) with iQ SYBR Green Supermix and specific primers. Every reaction was performed in triplicate. The PCR was performed as follows: $95^{\circ} \mathrm{C}$ for $5 \mathrm{~min}, 45$ cycles at $95^{\circ} \mathrm{C}$ for $20 \mathrm{~s}, 60^{\circ} \mathrm{C}$ for $20 \mathrm{~s}$, and $72^{\circ} \mathrm{C}$ for $20 \mathrm{~s}$. The relative expression value of each gene was calculated by the $\Delta \Delta \mathrm{Ct}$ method (Livak and Schmittgen 2001). The relative levels of the target transcripts were normalized to those of $18 S$ rRNA, Actin1, and $E F I \alpha$, which were included as internal controls.

\section{Subcellular localization of CabZIP2:GFP fusion protein.}

The coding region of the CabZIP2 gene without the stop codon was inserted into the GFP-fused binary vector p326GFP. Agrobacterium tumefaciens GV3101 carrying the construct was combined with the p19 strain (1:1 ratio; optical density at $\left.600 \mathrm{~nm}\left[\mathrm{OD}_{600}\right]=0.5\right)$ and was coinfiltrated into fully expanded leaves of 5-week-old $N$. benthamiana. For microscopic analyses, leaf discs were cut 2 days after infiltration. Cells of the lower epidermis were analyzed by the confocal laserscanning microscope LSM 700 (Carl Zeiss, Oberkochen, Germany) operated with LSM Image Browser software. For studying flg22-induced relocalization of CabZIP2 protein, $10 \mathrm{1M}$ flg22 peptide was infiltrated into leaves expressing CabZIP2-GFP at least $1 \mathrm{~h}$ before analysis.

\section{Transactivation analysis of CabZIP2 in yeast.}

The coding sequences for the full-length and truncated CabZIP2 mutants were cloned into the pGBKT7 vector (Clontech, Palo Alto, CA, U.S.A.), which contains a GAL4 DNA-binding domain. Transactivation by CabZIP2 was examined in Saccharomyces cerevisiae AH109, in which Ade1 and His3 reporter genes were driven by GALA promoters. Yeast cells were transformed with expression vectors that carried GALA-CabZIP2 fusion genes, as described in the manufacturer's instructions (Clontech, Palo Alto, CA, U.S.A.). After selection on SD (-Trp, -Leu) medium, the transformed yeast cells were transferred to SD (-Ade, - His, - Leu, -Trp) medium to confirm transcriptional activation.

\section{VIGS.}

The TRV-based VIGS system was used to knock down expression of the CabZIP2 gene in pepper plants (Kim and Hwang 2012). To silence $C a b Z I P 2$, a 254-bp fragment of CabZIP2 cDNA was inserted into the pTRV2 vector. Agrobacterium tumefaciens GV3101harboring pTRV1was combined with pTRV2:00 or
pTRV2:CabZIP2 (1:1 ratio) and was coinfiltrated into the fully expanded cotyledons of pepper plants $\left(\mathrm{OD}_{600}=0.2\right.$ for each construct). Plants were placed in a growth room under the same conditions as described above.

\section{BiFC analysis.}

To generate the BiFC constructs, full-length cDNAs with no stop codon of CabZIP2 were subcloned into 35S-SPYNE(R) 173 and 35S-SPYCE(M) vectors. For transient expression, Agrobacterium tumefaciens GV3101 strains harboring each construct were mixed with the p19 strain to avoid gene silencing and were infiltrated into the abaxial side of 5-week-old $N$. benthamiana leaves using a 1-ml needless syringe. For microscopic analyses, leaf discs were cut 4 days after infiltration. The lower epidermis cells were analyzed by confocal microscopy (model Zeiss 510 UV/Vis Meta) operated with LSM Image Browser software.

\section{Generation of the CabZIP2-OX transgenic plants.}

The full-length cDNA sequence of CabZIP2 was integrated into the pK2GW7 binary vector to induce constitutive expression of the CabZIP2 gene under the control of the Cauliflower mosaic virus (CaMV) 35S promoter in Arabidopsis. The CabZIP2 binary vectors were introduced into Agrobacterium tumefaciens GV3101 via electroporation. Agrobacteriummediated transformation of Arabidopsis thaliana with the CabZIP2 gene was carried out using the floral-dip method (Clough and Bent 1998). For selection of transgenic lines, seeds harvested from the putative transformed plants were sowed on MS agar plates containing $50 \mathrm{lg} \cdot$ of kanamycin per milliliter.

\section{ACKNOWLEDGMENTS}

This work was supported by a grant from the Next-Generation BioGreen 21 Program (number 01100501).

\section{LITERATURE CITED}

Adie, B. A., Pérez-Pérez, J., Pérez-Pérez, M. M., Godoy, M., SánchezSerrano, J. J., Schmelz, E. A., and Solano, R. 2007. ABA is an essential signal for plant resistance to pathogens affecting JA biosynthesis and the activation of defenses in Arabidopsis. Plant Cell 19:1665-1681.

Alazem, M., and Lin, N. S. 2014. Roles of plant hormones in the regulation of host-virus interactions. Mol. Plant Pathol. 16:529-540.

Alves, M. S., Dadalto, S. P., Gonçalves, A. B., De Souza, G. B., Barros, V. A., and Fietto, L. G. 2013. Plant bZIP Transcription factors responsive to pathogens: A review. Int. J. Mol. Sci. 14:7815-7828.

Baloglu, M. C., Eldem, V., Hajyzadeh, M., and Unver, T. 2014. Genomewide analysis of the bZIP transcription factors in cucumber. PLoS ONE 9:e96014.

Blanco, F. A., and Judelson, H. S. 2005. A bZIP transcription factor from Phytophthora interacts with a protein kinase and is required for zoospore motility and plant infection. Mol. Microbiol. 56:638-648.

Blée, E. 2002. Impact of phyto-oxylipins in plant defense. Trends Plant Sci. 7:315-322.

Boyle, P., and Després, C. 2010. Dual-function transcription factors and their entourage: Unique and unifying themes governing two pathogenesis-related genes. Plant Signal. Behav. 5:629-634.

Cantin, G. T., Stevens, J. L., and Berk, A. J. 2003. Activation domainmediator interactions promote transcription preinitiation complex assembly on promoter DNA. Proc. Natl. Acad. Sci. U.S.A. 100:12003-12008.

Chen, Z., Zheng, Z., Huang, J., Lai, Z., and Fan, B. 2009. Biosynthesis of salicylic acid in plants. Plant Signal. Behav. 4:493-496.

Clarke, J. D., Volko, S. M., Ledford, H., Ausubel, F. M., and Dong, X. 2000. Roles of salicylic acid, jasmonic acid, and ethylene in cpr-induced resistance in Arabidopsis. Plant Cell 12:2175-2190.

Clough, S. J., and Bent, A. F. 1998. Floral dip: A simplified method for Agrobacterium-mediated transformation of Arabidopsis thaliana. Plant J. 16:735-743.

Corrêa, L. G., Riaño-Pachón, D. M., Schrago, C. G., dos Santos, R. V., Mueller-Roeber, B., and Vincentz, M. 2008. The role of bZIP transcription factors in green plant evolution: Adaptive features emerging from four founder genes. PLoS ONE 3:e2944. 
Deppmann, C. D., Alvania, R. S., and Taparowsky, E. J. 2006. Cross-species annotation of basic leucine zipper factor interactions: Insight into the evolution of closed interaction networks. Mol. Biol. Evol. 23:1480-1492.

Després, C., Chubak, C., Rochon, A., Clark, R., Bethune, T., Desveaux, D., and Fobert, P. R. 2003. The Arabidopsis NPR1 disease resistance protein is a novel cofactor that confers redox regulation of DNA binding activity to the basic domain/leucine zipper transcription factor TGA1. Plant Cell 15:2181-2191.

Djamei, A., Pitzschke, A., Nakagami, H., Rajh, I., and Hirt, H. 2007. Trojan horse strategy in Agrobacterium transformation: Abusing MAPK defense signaling. Science 318:453-456.

Eulgem, T., Rushton, P. J., Robatzek, S., and Somssich, I. E. 2000. The WRKY superfamily of plant transcription factors. Trends Plant Sci. 5: 199-206.

Ferrari, S., Plotnikova, J. M., De Lorenzo, G., and Ausubel, F. M. 2003. Arabidopsis local resistance to Botrytis cinerea involves salicylic acid and camalexin and requires EDS4 and PAD2, but not SID2, EDS5 or PAD4. Plant J. 35:193-205.

Froidure, S., Canonne, J., Daniel, X., Jauneau, A., Brière, C., Roby, D., and Rivas, S. 2010. AtsPLA2- $\alpha$ nuclear relocalization by the Arabidopsis transcription factor AtMYB30 leads to repression of the plant defense response. Proc. Natl. Acad. Sci. U.S.A. 107:15281-15286.

Fu, Z. Q., and Dong, X. 2013. Systemic acquired resistance: Turning local infection into global defense. Annu. Rev. Plant Biol. 64:839-863.

Hahn, S. 1993. Structure(?) and function of acidic transcription activators. Cell 72:481-483.

Hong, J. K., Lee, S. C., and Hwang, B. K. 2005. Activation of pepper basic PR-1 gene promoter during defense signaling to pathogen, abiotic and environmental stresses. Gene 356:169-180.

Jakoby, M., Weisshaar, B., Dröge-Laser, W., Vicente-Carbajosa, J., Tiedemann, J., Kroj, T., and Parcy, F.; bZIP Research Group. 2002. bZIP transcription factors in Arabidopsis. Trends Plant Sci. 7:106-111.

Jones, J. D., and Dangl, J. L. 2006. The plant immune system. Nature 444: 323-329.

Jung, H. W., and Hwang, B. K. 2000. Isolation, partial sequencing, and expression of pathogenesis-related cDNA genes from pepper leaves infected by Xanthomonas campestris pv. vesicatoria. Mol. PlantMicrobe Interact. 13:136-142.

Kang, S. G., Price, J., Lin, P. C., Hong, J. C., and Jang, J. C. 2010. The Arabidopsis bZIP1 transcription factor is involved in sugar signaling, protein networking, and DNA binding. Mol. Plant 3:361-373.

Katagiri, F., Thilmony, R., and He, S. Y. 2002. The Arabidopsis thalianaPseudomonas syringae interaction. Arabidopsis Book 1:e0039.

Kesarwani, M., Yoo, J., and Dong, X. 2007. Genetic interactions of TGA transcription factors in the regulation of pathogenesis-related genes and disease resistance in Arabidopsis. Plant Physiol. 144:336-346.

Kim, H. S., and Delaney, T. P. 2002. Over-expression of TGA5, which encodes a bZIP transcription factor that interacts with NIM1/NPR1, confers SAR-independent resistance in Arabidopsis thaliana to Peronospora parasitica. Plant J. 32:151-163.

Kim, D. S., and Hwang, B. K. 2012. The pepper MLO gene, CaMLO2, is involved in the susceptibility cell-death response and bacterial and oomycete proliferation. Plant J. 72:843-855.

Lazebnik, J., Frago, E., Dicke, M., and van Loon, J. J. 2014. Phytohormone mediation of interactions between herbivores and plant pathogens. J. Chem. Ecol. 40:730-741

Lee, Y. K., and Hwang, B. K. 1996. Differential induction and accumulation of $\beta$-1,3-glucanase and chitinase isoforms in the intercellular space and leaf tissues of pepper by Xanthomonas campestris pv. vesicatoria infection. J. Phytopathol. 144:79-87.

Lee, S. C., and Hwang, B. K. 2009. Functional roles of the pepper antimicrobial protein gene, CaAMP1, in abscisic acid signaling, and salt and drought tolerance in Arabidopsis. Planta 229:383-391.

Lee, S. C., Choi, H. W., Hwang, I. S., Choi, D. S., and Hwang, B. K. 2006 a. Functional roles of the pepper pathogen-induced bZIP transcription factor, CAbZIP1, in enhanced resistance to pathogen infection and environmental stresses. Planta 224:1209-1225.
Lee, S. C., Kim, S. H., An, S. H., Yi, S. Y., and Hwang, B. K. 2006b. Identification and functional expression of the pepper pathogen-induced gene, $C A P I P 2$, involved in disease resistance and drought and salt stress tolerance. Plant Mol. Biol. 62:151-164.

Lee, S. C., Hwang, I. S., Choi, H. W., and Hwang, B. K. 2008. Involvement of the pepper antimicrobial protein CaAMP1 gene in broad spectrum disease resistance. Plant Physiol. 148:1004-1020.

Lee, S. C., Choi, D. S., Hwang, I. S., and Hwang, B. K. 2010. The pepper oxidoreductase CaOXR1 interacts with the transcription factor CaRAV1 and is required for salt and osmotic stress tolerance. Plant Mol. Biol. 73: 409-424.

Li, J. B., Luan, Y. S., and Yin, Y. L. 2014. SpMYB overexpression in tobacco plants leads to altered abiotic and biotic stress responses. Gene 547: $145-151$.

Lim, C. W., and Lee, S. C. 2014. Functional roles of the pepper MLO protein gene, CaMLO2, in abscisic acid signaling and drought sensitivity. Plant Mol. Biol. 85:1-10.

Lippok, B., Birkenbihl, R. P., Rivory, G., Brümmer, J., Schmelzer, E., Logemann, E., and Somssich, I. E. 2007. Expression of AtWRKY33 encoding a pathogen- or PAMP-responsive WRKY transcription factor is regulated by a composite DNA motif containing W box elements. Mol. Plant-Microbe Interact. 20:420-429.

Livak, K. J., and Schmittgen, T. D. 2001. Analysis of relative gene expression data using real-time quantitative PCR and the $2(-\Delta \Delta \mathrm{C}(\mathrm{T}))$ Method. Methods 25:402-408.

Llorca, C. M., Potschin, M., and Zentgraf, U. 2014. bZIPs and WRKYs: Two large transcription factor families executing two different functional strategies. Front. Plant Sci. 5:169.

Murashige, T., and Skoog, F. 1962. A revised medium for rapid growth and bio-assays with tobacco tissue cultures. Physiol. Plant. 15:473-497.

Pitzschke, A., Djamei, A., Teige, M., and Hirt, H. 2009. VIP1 response elements mediate mitogen-activated protein kinase 3-induced stress gene expression. Proc. Natl. Acad. Sci. U.S.A. 106:18414-18419.

Pogenberg, V., Consani Textor, L., Vanhille, L., Holton, S. J., Sieweke, M. H., and Wilmanns, M. 2014. Design of a bZip transcription factor with homo/heterodimer-induced DNA-binding preference. Structure 22:466-477.

Schwechheimer, C., Zourelidou, M., and Bevan, M. W. 1998a. Plant transcription factor studies. Annu. Rev. Plant Physiol. Plant Mol. Biol. 49:127-150.

Schwechheimer, C., Smith, C., and Bevan, M. W. 1998b. The activities of acidic and glutamine-rich transcriptional activation domains in plant cells: Design of modular transcription factors for high-level expression. Plant Mol. Biol. 36:195-204.

Sohn, K. H., Lee, S. C., Jung, H. W., Hong, J. K., and Hwang, B. K. 2006. Expression and functional roles of the pepper pathogen-induced transcription factor RAV1 in bacterial disease resistance, and drought and salt stress tolerance. Plant Mol. Biol. 61:897-915.

Thatcher, L. F., Kazan, K., and Manners, J. M. 2012. Lateral organ boundaries domain transcription factors: New roles in plant defense. Plant Signal. Behav. 7:1702-1704.

Wang, J., Zhou, J., Zhang, B., Vanitha, J., Ramachandran, S., and Jiang, S. Y. 2011. Genome-wide expansion and expression divergence of the basic leucine zipper transcription factors in higher plants with an emphasis on sorghum. J. Integr. Plant Biol. 53:212-231.

Whalen, M. C., Innes, R. W., Bent, A. F., and Staskawicz, B. J. 1991. Identification of Pseudomonas syringae pathogens of Arabidopsis and a bacterial locus determining avirulence on both Arabidopsis and soybean. Plant Cell 3:49-59.

Zhang, Y., Tessaro, M. J., Lassner, M., and Li, X. 2003. Knockout analysis of Arabidopsis transcription factors TGA2, TGA5, and TGA6 reveals their redundant and essential roles in systemic acquired resistance. Plant Cell 15:2647-2653.

Zhu, X., Qi, L., Liu, X., Cai, S., Xu, H., Huang, R., Li, J., Wei, X., and Zhang, Z. 2014. The wheat ethylene response factor transcription factor pathogen-induced ERF1 mediates host responses to both the necrotrophic pathogen Rhizoctonia cerealis and freezing stresses. Plant Physiol. 164:1499-1514. 